\title{
Fabrication Process and Thermoelectric Properties of $\mathrm{CNT} / \mathrm{Bi}_{2}(\mathrm{Se}, \mathrm{Te})_{3}$ Composites
}

\author{
Kyung Tae Kim, ${ }^{1}$ Yeong Seong Eom, ${ }^{1,2}$ and Injoon Son ${ }^{2}$ \\ ${ }^{1}$ Powder \& Ceramic Materials Division, Korea Institute of Materials Science, 797 Changwon-daero, Seongsan-gu, \\ Changwon, Gyeongnam 642-831, Republic of Korea \\ ${ }^{2}$ Department of Materials Science and Metallurgical Engineering, Kyungpook National University, 80 Daehakro, Buk-gu, \\ Daegu, 702-701, Republic of Korea \\ Correspondence should be addressed to Kyung Tae Kim; ktkim@kims.re.kr
}

Received 17 November 2014; Accepted 28 December 2014

Academic Editor: Kyu Hyoung Lee

Copyright (C) 2015 Kyung Tae Kim et al. This is an open access article distributed under the Creative Commons Attribution License, which permits unrestricted use, distribution, and reproduction in any medium, provided the original work is properly cited.

Carbon nanotube/bismuth-selenium-tellurium composites were fabricated by consolidating $\mathrm{CNT} / \mathrm{Bi}_{2}(\mathrm{Se}, \mathrm{Te})_{3}$ composite powders prepared from a polyol-reduction process. The synthesized composite powders exhibit CNTs homogeneously dispersed among $\mathrm{Bi}_{2}(\mathrm{Se}, \mathrm{Te})_{3}$ matrix nanopowders of $300 \mathrm{~nm}$ in size. The powders were densified into a CNT/Bi ${ }_{2}(\mathrm{Se}, \mathrm{Te})_{3}$ composite in which CNTs were randomly dispersed in the matrix through spark plasma sintering process. The effect of an addition of Se on the dimensionless figure-of-merit (ZT) of the composite was clearly shown in 3 vol.\% $\mathrm{CNT} / \mathrm{Bi}_{2}(\mathrm{Se}, \mathrm{Te})_{3}$ composite as compared to $\mathrm{CNT} / \mathrm{Bi}_{2} \mathrm{Te}_{3}$ composite throughout the temperature range of 298 to $473 \mathrm{~K}$. These results imply that matrix modifications such as an addition of Se as well as the incorporation of CNTs into bismuth telluride thermoelectric materials is a promising means of achieving synergistic enhancement of the thermoelectric performance levels of these materials.

\section{Introduction}

Thermoelectric (TE) materials have strong potential as novel power generators and as coolers or heaters because they can directly convert temperature gradients into electricity and vice versa in a solid state $[1,2]$. In order to achieve high energy conversion efficiency, dimensionless figure-of-merit, $\mathrm{ZT}=T\left(\alpha^{2} / \rho \kappa\right)$, in which $\alpha$ is the Seebeck coefficient, $\rho$ and $\kappa$ are, respectively, the electrical resistivity and thermal conductivity, and $T$ is the absolute temperature, should be increased.

Amongst many TE materials, alloys based on bismuth telluride $\left(\mathrm{Bi}_{2} \mathrm{Te}_{3}\right)$ have shown superior $\mathrm{ZT}$ values at temperatures ranging from ambient temperatures to $500 \mathrm{~K}$ due to their feasible combination of high electrical and low thermal conductivity [2-4]. Due to the wide variety of practical applications, many researchers have attempted to enhance the TE properties of $\mathrm{Bi}_{2} \mathrm{Te}_{3}$ materials. From the viewpoint of microstructural engineering, as means of independently controlling the electrical conductivity and thermal conductivity, the formation of new grain boundaries and interfaces is a viable means of promoting active phonon scattering, resulting in extremely low thermal conductivity. Recently, it was reported that a TE nanocomposite with an addition of nanoparticles into the TE matrix offers many of these properties because the extrinsic interface created by the newly added nanoparticles and the intrinsic grain boundaries can produce high electrical properties as well as low thermal conductivity [5-7].

In the past few decades, CNTs have become very wellknown nanomaterials capable of supplying a large interfacial area when they are homogeneously dispersed in a matrix. More recently, it was reported that CNTs can be utilized to improve the $\mathrm{ZT}$ values of pure $\mathrm{Bi}_{2} \mathrm{Te}_{3}$ materials by inducing a considerable degree of large lattice phonon scattering $[3,8,9]$. Additionally, performance-enhancements of p-type Bi-SbTe based materials have been rigorously investigated. Thus far, however, there have been few results based on n-type $\mathrm{Bi}_{2} \mathrm{Te}_{3}$ materials by matrix modification such as Selenium (Se) doping. 
In this study, we fabricate $\mathrm{CNT} / \mathrm{Bi}_{2}(\mathrm{Se}, \mathrm{Te})_{3}$ composite powders using a polyol-reduction process of Bi salt, Te salt, and Se salt in which 3 vol. $\%$ of CNTs with a multiwalled structure are randomly dispersed in n-type $\mathrm{Bi}_{2}(\mathrm{Se}, \mathrm{Te})_{3}$ matrix powders, leading to improved thermoelectric properties of the composite. In order to verify the effect of the addition of $\mathrm{Se}, \mathrm{CNT} / \mathrm{Bi}_{2} \mathrm{Te}_{3}$ composite is also fabricated via the same process but without Se.

\section{Experimental Procedure}

For the fabrication of the $\mathrm{CNT} / \mathrm{Bi}_{2}(\mathrm{Se}, \mathrm{Te})_{3}$ composite, multiwalled CNTs prepared from a thermal CVD process $(98 \%$ purity, Hanwha Nanotech Co. Ltd.) were utilized. The average length and diameter of the CNTs are about $2 \sim 3 \mu \mathrm{m}$ and $20 \mathrm{~nm}$, respectively. The CNTs used in this study were chemically treated to attach oxygen-contained functional groups, in this case carbonyl or carboxyl groups, on their surfaces. The functional groups thus formed play an important role as intermediate materials to bond the $\mathrm{Bi}, \mathrm{Se}$, and $\mathrm{Te}$ atoms to the CNTs. (More detailed and additional experimental techniques are described in the literature [10, 11].) The functionalized CNTs were finally centrifuged and dried in a vacuum at $10^{-2}$ torr. $2 \mathrm{mg}$ of the functionalized CNTs, $9 \mathrm{~mL}$ of oleylamine, and $4.5 \mathrm{~mL}$ of 1-dodecanthiol were added to the reactor and mixed as a CNT dispersion solution. Next, $0.154 \mathrm{~g}$ of $\mathrm{Bi}\left(\mathrm{CH}_{3} \mathrm{COO}\right)_{3}, 0.145 \mathrm{~g}$ of $\mathrm{TeCl}_{4}$, and $0.013 \mathrm{~g}$ of $\mathrm{SeCl}_{4}$ as metal precursors for the $\mathrm{Bi}_{2}(\mathrm{Se}, \mathrm{Te})_{3}$ composition were added to the CNT solution and an ultrasonic treatment was applied to mix the solution.

The mixed solution was then heated to $110^{\circ} \mathrm{C}$ for 2 hours under an argon gas atmosphere. During the chemical reaction process, $\mathrm{Bi}$, Te, and $\mathrm{Se}$ ions dissolved from each salt were reduced to $\mathrm{Bi}, \mathrm{Te}$, and Se atoms by 1 -dodecanthiol, after which Bi-Se-Te powders were heterogeneously nucleated and grown onto the surfaces of the CNTs. After this chemical reaction, the newly formed powders were cleansed with the solvent hexane and collected by a centrifugation process. The synthesized powders were consolidated by means of the spark plasma sintering (SPS) process (Sumitomo, Dr. SinterLab). The sintering condition of the powders was $623 \mathrm{~K}$ for $10 \mathrm{~min}$ in a vacuum at $10^{-3}$ torr with an applied pressure of $50 \mathrm{MPa}$. The volume percent of CNTs in the powders was calculated from the carbon contents as evaluated in an elemental analysis (EA1110-FISONS) of the fabricated CNT/Bi-Se-Te powders. It was found that an amount of $1.0 \mathrm{wt} \% \mathrm{CNTs}$ is similar to 3 vol.\% of CNTs in Bi-Se-Te powders considering the volume of $2.0 \mathrm{~g} / \mathrm{cm}^{3}$ of multiwalled CNTs.

The microstructures of CNT/Bi-Se-Te composite powders and the composite material were analyzed by fieldemission scanning electron microscopy (FESEM, MIRA II LMH, Tescan) and transmission electron microscopy (TEM, $200 \mathrm{kV}$, JEM2100F, JEOL). The phases of the composite powders were investigated by X-ray diffraction (XRD) using $\mathrm{Cu} / \mathrm{K} \alpha$ radiation $(\lambda=1.54056 \AA)$. The Seebeck coefficient and electrical conductivity were evaluated with a four-probe method (Ulvac-Rico, ZEM-3) at different temperatures ranging from $298 \mathrm{~K}$ to $473 \mathrm{~K}$. The measured thermal diffusivity (laser flash method, LFA457, NETZSCH) was calculated with the thermal conductivity using the specific heat obtained from a differential scanning calorimeter (DSC) and the density of the sintered CNT/Bi-Se-Te composites.

\section{Results and Discussion}

Figure 1(a) shows the surface morphology of the synthesized $\mathrm{CNT} / \mathrm{Bi}-\mathrm{Se}-\mathrm{Te}$ composite powders, where the CNTs are randomly dispersed in the $\mathrm{Bi}-\mathrm{Se}-\mathrm{Te}$ matrix nanopowders. The CNTs are not severely agglomerated but are instead individually dispersed at the matrix powders. The phase of the $\mathrm{Bi}$-Te-Se matrix is clearly confirmed as $\mathrm{Bi}_{2}(\mathrm{Se}, \mathrm{Te})_{3}$ as shown in the XRD patterns in Figure 1(b), corresponding to ICDD PDF number 50-0954, showing composition of $\mathrm{Bi}_{2} \mathrm{Se}_{0.3} \mathrm{Te}_{2.7}$. The TEM image indicates that one CNT is bonded with several $\mathrm{Bi}_{2}(\mathrm{Se}, \mathrm{Te})_{3}$ nanopowders as shown in Figure $1(\mathrm{c})$. The CNT appears to have penetrated or been implanted into the matrix nanopowders. The high-resolution TEM image of the composite powders shown in Figure 1(d) indicates that the structure of the multiwalled CNT is retained even after the chemical reaction process.

Figure 2(a) shows the fracture surface of sintered $\mathrm{CNT} / \mathrm{Bi}_{2}(\mathrm{Se}, \mathrm{Te})_{3}$ composite. The SEM image reveals that several CNTs are implanted onto $\mathrm{Bi}_{2}(\mathrm{Se}, \mathrm{Te})_{3}$ matrix grains with faceted shape. It was verified that there was no severe agglomeration of the CNTs on the surface of the composite. Interestingly, the fracture surface image is very similar to that of a $\mathrm{CNT} / \mathrm{Bi}_{2} \mathrm{Te}_{3}$ composite fabricated by the same process for comparison but without an addition of Se, as shown in Figure 2(b). The CNTs were found to be randomly dispersed in the matrix, as displayed in Figure 2(c). The high-resolution TEM image shown in the white box in Figure 2(c) confirms that the embedded CNTs also had multiwalled carbon nanotubes like the composite powders but without secondary phase at the interface between the CNT and the $\mathrm{Bi}_{2}(\mathrm{Se}, \mathrm{Te})_{3}$ matrix region (Figure $2(\mathrm{~d})$ ). Our previous work reported that only interfacial oxygen atoms supplied from oxygen-contained functional groups may exist at the interfacial region to bond CNT to the Bi-Te materials [10].

The relative density and Hall-effect measurements of pure $\mathrm{Bi}_{2} \mathrm{Te}_{3}, \mathrm{CNT} / \mathrm{Bi}_{2} \mathrm{Te}_{3}$, and $\mathrm{CNT} / \mathrm{Bi}_{2}(\mathrm{Se}, \mathrm{Te})_{3}$ composites characterized at the room temperature are displayed in Table 1. The relative densities of all samples exceeded $98 \%$ indicating that they are similarly densified with low porosity levels. The measured electrical resistivity increased from $0.92 \times 10^{-5} \Omega \mathrm{m}$ to $1.24 \times 10^{-5} \Omega \mathrm{m}$ upon the addition of Se materials. The CNTs also increased the electrical resistivity of the $\mathrm{Bi}_{2} \mathrm{Te}_{3}$ materials due to their low carrier mobility and low carrier concentration. This result implies that the embedded CNTs in this study show p-type semiconducting behaviors. That is, it seems that p-type carriers stemming from CNTs offset concentration of $n$-type carriers in $\mathrm{Bi}_{2} \mathrm{Te}_{3}$ matrix. Also, it is well known that electrical conductivity $(\sigma)$ is directly proportional to carrier density $(n)$, electronic charge $(e)$, and carrier mobility $(\mu)$ as follows:

$$
\sigma=n e \mu .
$$




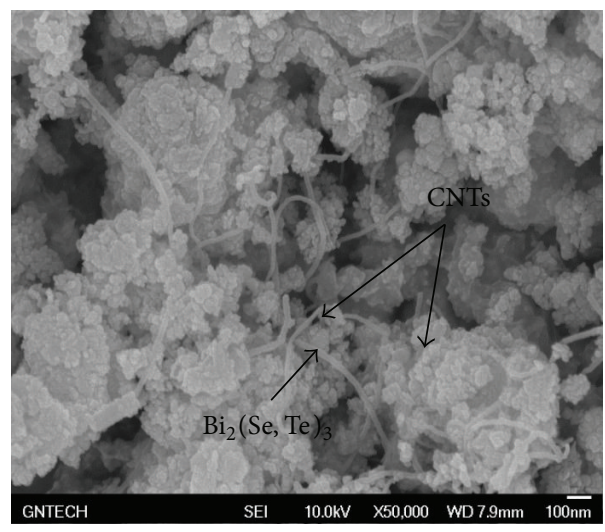

(a)

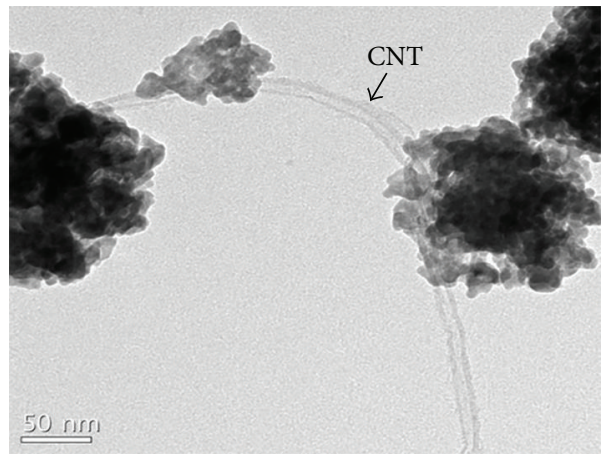

(c)

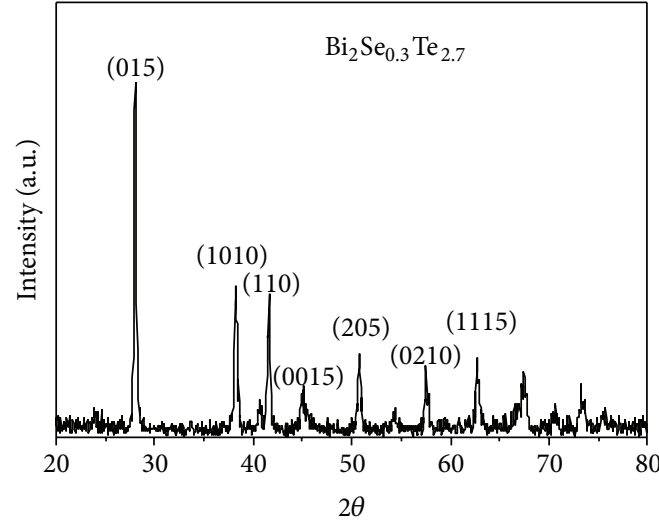

(b)



(d)

FIgure 1: (a) Surface SEM image, (b) X-ray diffraction pattern, (c) TEM image, and (d) high-resolution TEM image of the synthesized $\mathrm{CNT} / \mathrm{Bi}_{2}(\mathrm{Se}, \mathrm{Te})_{3}$ composite powders.

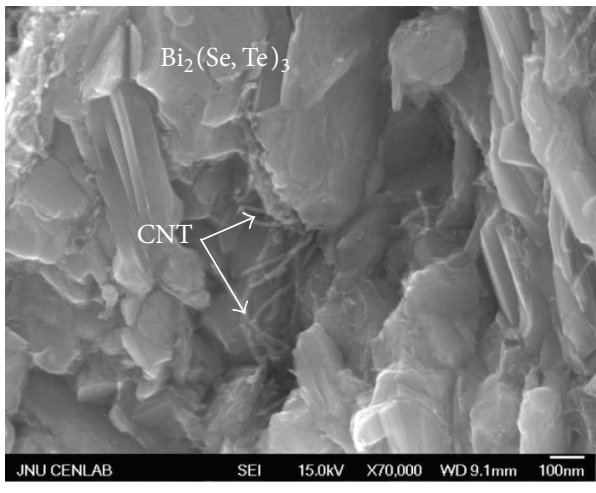

(a)

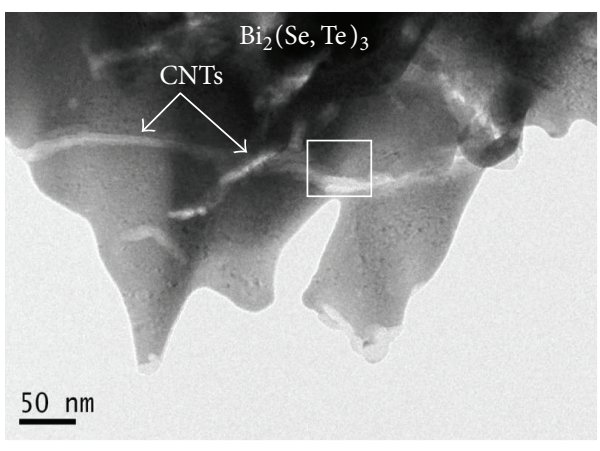

(c)



(b)

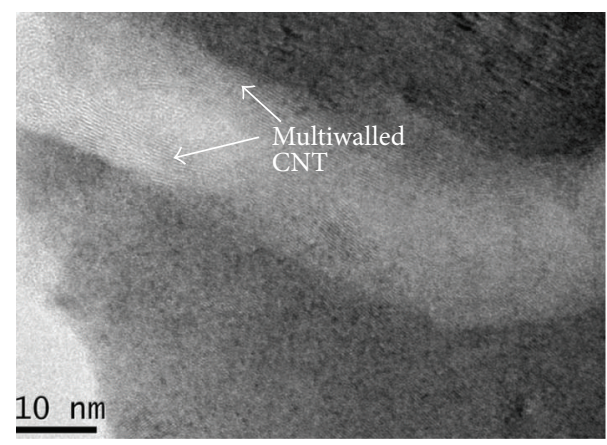

(d)

FIGURE 2: (a) FE-SEM image of the fracture surface of the CNT/Bi ${ }_{2}(\mathrm{Se}, \mathrm{Te})_{3}$ composite, (b) FE-SEM image of a CNT/Bi $\mathrm{Te}_{3}$ composite fabricated for the comparison, (c) TEM image exhibiting the dispersion state of the CNTs, and (d) a high-resolution TEM image showing multiwalled structured CNT present in the matrix. 
TABLE 1: Comparison of the relative density levels, electric resistivity levels, carrier concentrations, carrier mobility levels, and Seebeck coefficients.

\begin{tabular}{lccccc}
\hline & $\begin{array}{c}\text { Relative density } \\
(\%)\end{array}$ & $\begin{array}{c}\text { Electrical resistivity } \\
\left(10^{-5} \Omega \cdot \mathrm{m}\right)\end{array}$ & $\begin{array}{c}\text { Carrier mobility } \\
\left(\mathrm{cm}^{2} / \mathrm{Vs}\right)\end{array}$ & $\begin{array}{c}\text { Carrier concentration } \\
\left(/ \mathrm{cm}^{3}\right)\end{array}$ & $\begin{array}{c}\text { Seebeck coefficient } \\
(\mu \mathrm{V} / \mathrm{K})\end{array}$ \\
\hline $\mathrm{Bi}_{2} \mathrm{Te}_{3}$ & $98 \pm 0.3$ & 0.78 & 250 & $-8.1 \times 10^{19}$ & -83 \\
$\mathrm{CNT} / \mathrm{Bi}_{2} \mathrm{Te}_{3}$ & $98 \pm 0.3$ & 0.92 & 155 & $-7.5 \times 10^{18}$ & -106 \\
$\mathrm{CNT} / \mathrm{Bi}_{2}(\mathrm{Se}, \mathrm{Te})_{3}$ & $98 \pm 0.5$ & 1.24 & 138 & $-3.1 \times 10^{18}$ & -122 \\
\hline
\end{tabular}



(a)

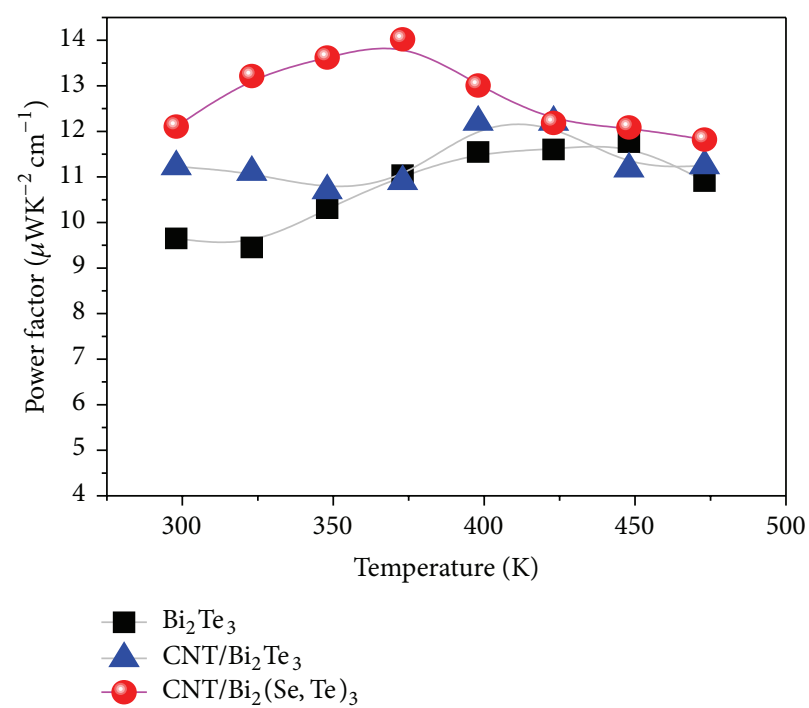

(c)

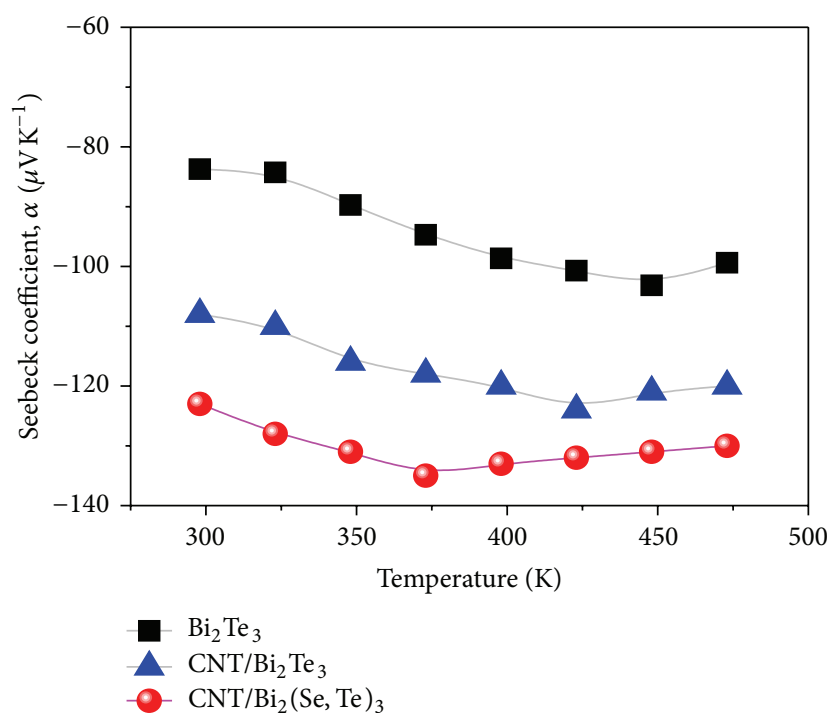

(b)



(d)

FIgURE 3: Comparison of the thermoelectric properties of pure $\mathrm{Bi}_{2} \mathrm{Te}_{3}$, the $\mathrm{CNT} / \mathrm{Bi}_{2} \mathrm{Te}_{3}$ composite, and the CNT/Bi ${ }_{2}(\mathrm{Se}, \mathrm{Te})_{3}$ composite: (a) electrical resistivity, (b) Seebeck coefficient, (c) power factor, and (d) thermal conductivity.

Because carrier concentration and carrier mobility decrease by addition of CNT, respectively, electrical resistivity increases. Furthermore, given the results shown in Table 1 and Figure 3(a), it can be assumed that the many interfaces generated from the CNTs dispersed in the $\mathrm{Bi}_{2} \mathrm{Te}_{3}$ matrix along with Se acting as an alloying agent result in a reduction of the carrier mobility.

Figure 3(a) shows the variation in the electrical resistivity levels of all samples as a function of the temperature when it ranges from $298 \mathrm{~K}$ to $473 \mathrm{~K}$. The $\mathrm{CNT} / \mathrm{Bi}_{2}(\mathrm{Se}, \mathrm{Te})_{3}$ composite 
shows higher electrical resistivity throughout the temperature range. This likely originated from carrier scattering at the newly formed interfaces created by the CNT addition and from the alloying effect caused by Se doping. At the same time, this is also proven by the lowest carrier concentration, $-3.1 \times 10^{18} / \mathrm{cm}^{3}$ for $\mathrm{CNT} / \mathrm{Bi}_{2}(\mathrm{Se}, \mathrm{Te})_{3}$ compared to those of the $\mathrm{Bi}_{2} \mathrm{Te}_{3}$ and $\mathrm{CNT} / \mathrm{Bi}_{2} \mathrm{Te}_{3}$ samples. Figure $3(\mathrm{~b})$ shows the Seebeck coefficients measured in the same temperature range. Unlike the relationship between the electrical conductivity and the carrier concentration of (1), the Seebeck coefficient typically increases with a decrease in the carrier concentration. The Seebeck coefficient $\alpha$ is determined by

$$
\alpha=\frac{8 \pi^{2} \kappa_{B}^{2}}{3 e h^{2}} m^{*} T\left(\frac{\pi}{3 n}\right)^{2 / 3} .
$$

Here, $\kappa_{B}, e, h$, and $m^{*}$ are the Boltzmann constant, electronic charge, Planck constant, effective mass of the carrier, and the carrier concentration, respectively. Hence, The highest Seebeck coefficient of $-138 \mu \mathrm{V} / \mathrm{K}$ was obtained from the $3 \mathrm{vol} \% \mathrm{CNT} / \mathrm{Bi}_{2}(\mathrm{Se}, \mathrm{Te})_{3}$ composite at $373 \mathrm{~K}$.

Figure 3(c) shows the power factor values as calculated from the electrical resistivity values and the Seebeck coefficients. Although the power factor of $\mathrm{CNT} / \mathrm{Bi}_{2} \mathrm{Te}_{3}$ is somewhat higher than that of $\mathrm{Bi}_{2} \mathrm{Te}_{3}$ in the temperature ranges of 298$350 \mathrm{~K}$, similar values are observed over $350 \mathrm{~K}$. Comparing both samples, the $\mathrm{CNT} / \mathrm{Bi}_{2}(\mathrm{Se}, \mathrm{Te})_{3}$ composite shows a superior power factor until the temperature reaches $423 \mathrm{~K}$ due to the increased Seebeck coefficient at this temperature. According to (1) and (2), as carrier concentration decreases, Seebeck coefficient increases but electrical conductivity decreases. Thus, it can be described that the highest power factor value of the $\mathrm{CNT} / \mathrm{Bi}_{2}(\mathrm{Se}, \mathrm{Te})_{3}$ composite originates from the combination between Seebeck coefficient and electrical resistivity that varies with carrier concentration.

Figure 3(d) shows the thermal conductivity values of the $\mathrm{CNT} / \mathrm{Bi}_{2}(\mathrm{Se}, \mathrm{Te})_{3}$ composite compared to those of pure $\mathrm{Bi}_{2} \mathrm{Te}_{3}$ and the $\mathrm{CNT} / \mathrm{Bi}_{2} \mathrm{Te}_{3}$ composite as a function of the temperature when it ranges from $293 \mathrm{~K}$ to $473 \mathrm{~K}$. This result shows that a similarly significant reduction of the total thermal conductivity in the CNT composite regardless of the addition of Se is achieved. Hence, the reduction in the thermal conductivity may have originated from the lattice phonon and carrier scattering at the newly formed CNT/matrix interfaces and at the matrix grain boundaries, as reported previously by the authors for CNT or alumina nanoparticle $/ \mathrm{Bi}_{2} \mathrm{Te}_{3}$ systems [12]. Figure 4 indicates the lattice thermal conductivities, $\kappa_{L}$, as a function of temperature. The electrical contribution of total thermal conductivity, $\kappa$, was calculated from $\kappa_{\mathrm{el}}=L \sigma T$. Here, $L$ is Lorentz constant $\left(1.5 \times 10^{-8} \mathrm{~V}^{2} \mathrm{~K}^{-2}\right), \sigma$ is electrical conductivity, and $T$ is temperature. The lattice thermal conductivity was obtained by $\kappa-\kappa_{\mathrm{el}}$. As shown in Figure 4, $\mathrm{CNT} / \mathrm{Bi}_{2}(\mathrm{Se}, \mathrm{Te})_{3}$ composite shows lower $\kappa_{L}$ compared to the other two samples except temperature ranges of 298-323 K. This means that the addition of both CNT and Se actively generates lattice-phonon scattering resulting in low thermal conductivity. Furthermore, it was noted that the reduced value of the composites is closely related to the thermal

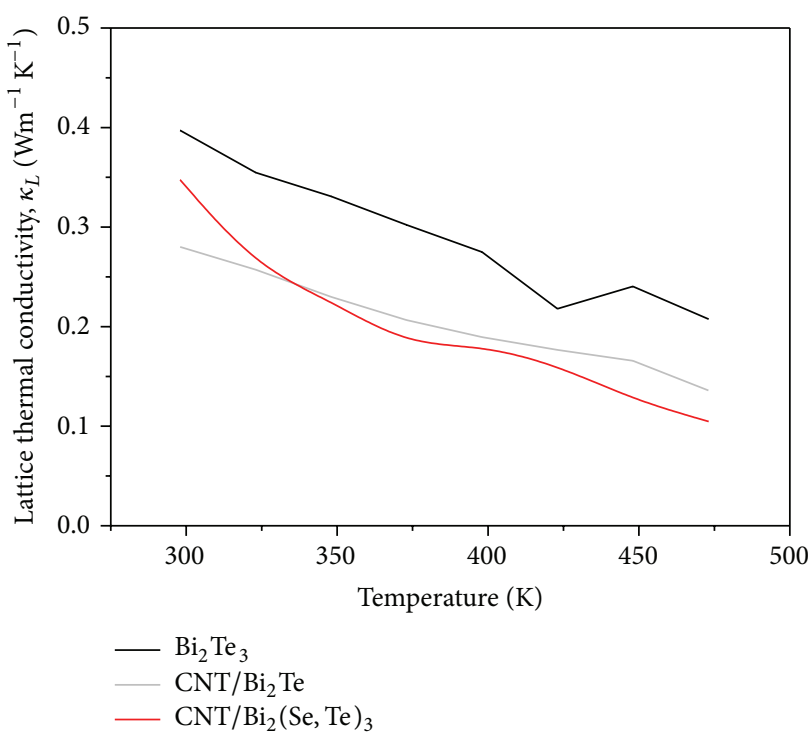

FIGURE 4: Comparison of the lattice thermal conductivity of pure $\mathrm{Bi}_{2} \mathrm{Te}_{3}$, the $\mathrm{CNT} / \mathrm{Bi}_{2} \mathrm{Te}_{3}$ composite, and the $\mathrm{CNT} / \mathrm{Bi}_{2}(\mathrm{Se}, \mathrm{Te})_{3}$ composite as a function of the temperature.

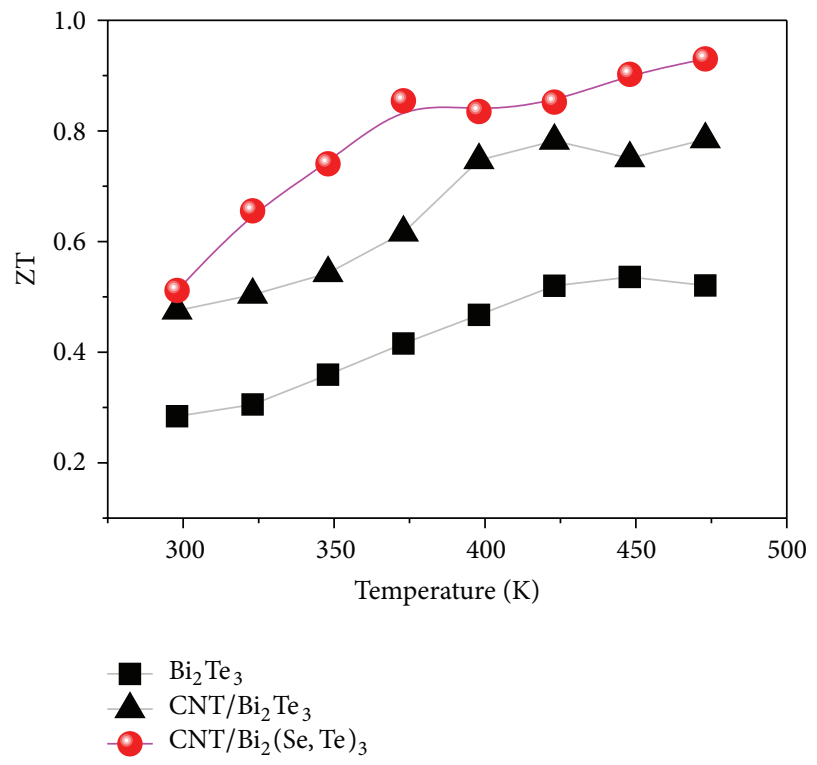

FIGURE 5: Comparison of the dimensionless figures-of-merit, ZT, of pure $\mathrm{Bi}_{2} \mathrm{Te}_{3}$, the $\mathrm{CNT} / \mathrm{Bi}_{2} \mathrm{Te}_{3}$ composite, and the $\mathrm{CNT} / \mathrm{Bi}_{2}(\mathrm{Se}, \mathrm{Te})_{3}$ composite as a function of the temperature.

resistance at the closed pores remaining after the sintering process.

Figure 5 shows a comparison of the dimensionless figures-of-merit (ZT) of $\mathrm{Bi}_{2} \mathrm{Te}_{3}, \mathrm{CNT} / \mathrm{Bi}_{2} \mathrm{Te}_{3}$, and $\mathrm{CNT} /$ $\mathrm{Bi}_{2}(\mathrm{Se}, \mathrm{Te})_{3}$ composites as calculated from the thermoelectric properties. The $\mathrm{ZT}$ values of the $\mathrm{CNT} / \mathrm{Bi}_{2}(\mathrm{Se}, \mathrm{Te})_{3}$ composite were found to be higher than those of $\mathrm{Bi}_{2} \mathrm{Te}_{3}$ and the $\mathrm{CNT} / \mathrm{Bi}_{2} \mathrm{Te}_{3}$ composite, regardless of the temperature. The peak ZT, 0.9 , of the 3 vol.\% $\mathrm{CNT} / \mathrm{Bi}_{2}(\mathrm{Se}, \mathrm{Te})_{3}$ composite was obtained at $473 \mathrm{~K}$. This absolute value is not remarkable 
but it is comparable to commercial n-type bismuth telluride materials despite the fact that the composite was synthesized by a chemical process. Nonetheless, these results clearly confirm that a modification of the matrix as well as an addition of CNTs should be considered to realize $\mathrm{Bi}_{2} \mathrm{Te}_{3}$ thermoelectric materials with high $\mathrm{ZT}$ values.

\section{Conclusions}

A CNT/Bi ${ }_{2}(\mathrm{Se}, \mathrm{Te})_{3}$ composite was fabricated by the spark plasma sintering of $\mathrm{CNT} / \mathrm{Bi}_{2}(\mathrm{Se}, \mathrm{Te})_{3}$ composite powders prepared from a polyol chemical reaction. The synthesized composite powders showed a random dispersion of CNTs among $\mathrm{Bi}_{2}(\mathrm{Se}, \mathrm{Te})_{3}$ matrix nanopowders without severe agglomeration. The consolidated $\mathrm{CNT} / \mathrm{Bi}_{2}(\mathrm{Se}, \mathrm{Te})_{3}$ composite shows superior thermoelectric properties, with higher Seebeck coefficients than those of the $\mathrm{CNT} / \mathrm{Bi}_{2} \mathrm{Te}_{3}$ composite along with low thermal conductivity. Hence, the results of this study demonstrate that matrix modification such as doping with Se in this case can be applied to enhance the thermoelectric properties of CNT-dispersed TE materials.

\section{Conflict of Interests}

The authors declare that there is no conflict of interests regarding the publication of this paper.

\section{Acknowledgments}

This work was supported by a Grant (code no. NRF2014063704) from the Global Frontier Research Program of the Ministry of Science, ICT \& Future Planning of the Republic of Korea.

\section{References}

[1] A. J. Minnich, M. S. Dresselhaus, Z. F. Ren, and G. Chen, "Bulk nanostructured thermoelectric materials: current research and future prospects," Energy and Environmental Science, vol. 2, no. 5, pp. 466-479, 2009.

[2] B. Poudel, Q. Hao, Y. Ma et al., "High-thermoelectric performance of nanostructured bismuth antimony telluride bulk alloys," Science, vol. 320, no. 5876, pp. 634-638, 2008.

[3] H. J. Goldsmid, "Bismuth telluride and its alloys as materials for thermoelectric generation," Materials, vol. 7, no. 4, pp. 25772592, 2014

[4] W. Xie, X. Tang, Y. Yan, Q. Zhang, and T. M. Tritt, "Unique nanostructures and enhanced thermoelectric performance of melt-spun BiSbTe alloys," Applied Physics Letters, vol. 94, no. 10, Article ID 102111, 2009.

[5] R. J. Mehta, Y. Zhang, C. Karthik et al., "A new class of doped nanobulk high-figure-of-merit thermoelectrics by scalable bottom-up assembly," Nature Materials, vol. 11, no. 3, pp. 233-240, 2012.

[6] L. P. Bulat, I. A. Drabkin, V. V. Karatayev et al., "The influence of anisotropy and nanoparticle size distribution on the lattice thermal conductivity and the thermoelectric figure of merit of nanostructured $(\mathrm{Bi}, \mathrm{Sb})_{2} \mathrm{Te}_{3}$," Journal of Electronic Materials, vol. 43, no. 6, pp. 2121-2126, 2014.
[7] J. Li, Q. Tan, J. F. Li et al., "BiSbTe-based nanocomposites with high ZT: the effect of SiC nanodispersion on thermoelectric properties," Advanced Functional Materials, vol. 23, no. 35, pp. 4317-4323, 2013.

[8] X. A. Fan, J. Y. Yang, R. G. Chen, W. Zhu, and S. Q. Bao, "Phase transformation and thermoelectric properties of p-type $\left(\mathrm{Bi}_{2} \mathrm{Te}_{3}\right)_{0.25}\left(\mathrm{Sb}_{2} \mathrm{Te}_{3}\right)_{0.75}$ prepared by mechanical alloying and hot pressing," Materials Science and Engineering A, vol. 438440, pp. 190-193, 2006.

[9] J. Jiang, L. Chen, S. Bai, Q. Yao, and Q. Wang, “Thermoelectric properties of textured p-type $(\mathrm{Bi}, \mathrm{Sb})_{2} \mathrm{Te}_{3}$ fabricated by spark plasma sintering," Scripta Materialia, vol. 52, no. 5, pp. 347-351, 2005.

[10] K. T. Kim, S. Y. Choi, E. H. Shin et al., "The influence of CNTs on the thermoelectric properties of a CNT/ $\mathrm{Bi}_{2} \mathrm{Te}_{3}$ composite," Carbon, vol. 52, pp. 541-549, 2013.

[11] K. T. Kim, D.-W. Kim, and G. H. Ha, "Direct synthesis of Te/ $\mathrm{Bi}_{2} \mathrm{Te}_{3}$ nanocomposite powders by a polyol process," Research on Chemical Intermediates, vol. 36, no. 6-7, pp. 835-841, 2010.

[12] K. T. Kim, H. Y. Koo, G.-G. Lee, and G. H. Ha, "Synthesis of alumina nanoparticle-embedded-bismuth telluride matrix thermoelectric composite powders," Materials Letters, vol. 82, pp. 141-144, 2012. 



Submit your manuscripts at http://www.hindawi.com
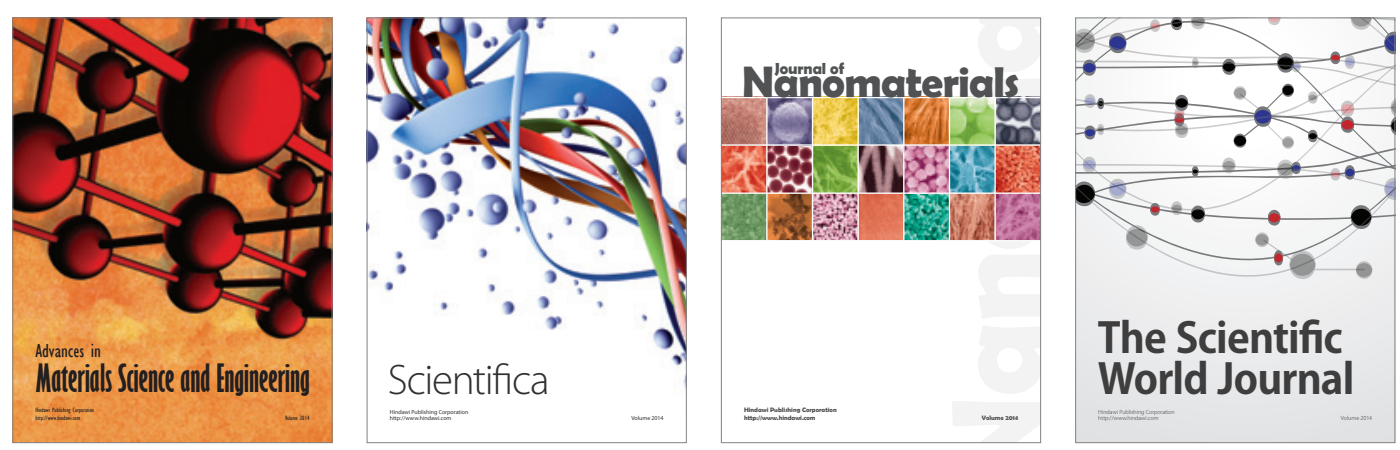

\section{The Scientific World Journal}
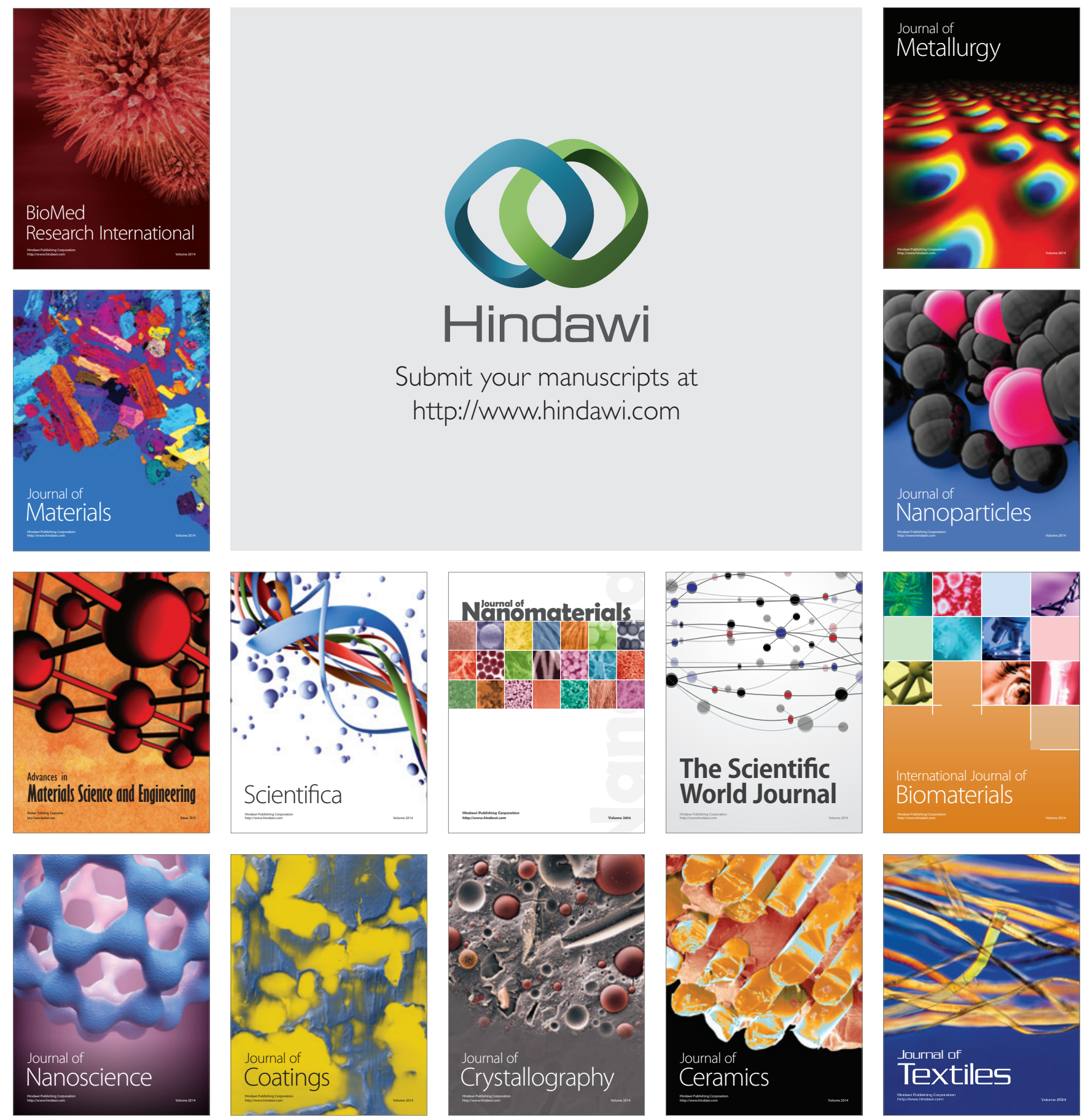